PROCEEDINGS OF THE

AMERICAN MATHEMATICAL SOCIETY

Volume 129, Number 8, Pages 2483-2487

S 0002-9939(01)05825-7

Article electronically published on January 18, 2001

\title{
REAL GROUPS TRANSITIVE ON COMPLEX FLAG MANIFOLDS
}

\author{
JOSEPH A. WOLF
}

(Communicated by Rebecca A. Herb)

\begin{abstract}
Let $Z=G / Q$ be a complex flag manifold. The compact real form $G_{u}$ of $G$ is transitive on $Z$. If $G_{0}$ is a noncompact real form, such transitivity is rare but occasionally happens. Here we work out a complete list of Lie subgroups of $G$ transitive on $Z$ and pick out the cases that are noncompact real forms of $G$.
\end{abstract}

\section{THE PROBLEM}

Let $Z=G / Q$ be a complex flag manifold where $G$ is a complex connected semisimple Lie group and $Q$ is a parabolic subgroup. Let $G_{0}$ be a real form of $G$. If $G_{0}$ is the compact real form, then it is transitive on $Z$. On a number of occasions the question has come up as to whether any noncompact real form of $G$ can be transitive on $Z$. Here I'll record the answer. The rough answer is "yes, but just a few." The precise answer, Corollaries 1.7 and 2.3 below, follows from a more general classification, Theorems 1.6 and 2.2. This more general classification uses a technique of D. Montgomery [M], together with some results of [W1] that depend in an essential way on a classification O1 of A. L. Onishchik.

After this paper was written I learned of Onishchik's book O2]. There is some overlap for compact groups, but there are no inclusions.

\section{The SOLUTION FOR IRREDUCIBLE FLAGS}

We formulate the problem in terms of transitive subgroups. Let $G_{u}$ be the compact real form of $G$, so $Z=G_{u} /\left(G_{u} \cap Q\right)$ and $G_{u} \cap Q$ is the compact real form of the reductive part of $Q$. Let $A \subset G$ be a closed subgroup that is transitive on $Z$. The identity component $A^{0}$ of $A$ is transitive on $Z$, because $Z$ is connected, so a maximal compact subgroup $B^{0} \subset A^{0}$ already is transitive on $Z$, according to Montgomery [M]. We may replace $A$ by a conjugate and assume $B=A \cap G_{u}$. So

Received by the editors July 28, 1999 and, in revised form, December 9, 1999.

2000 Mathematics Subject Classification. Primary 22E15; Secondary 22E10, 32E30, 32M10.

Key words and phrases. Semisimple Lie group, semisimple Lie algebra, representation, flag manifold, flag domain.

The author's research was supported by the Alexander von Humboldt Foundation and by NSF Grant DMS 97-05709. The author thanks the Ruhr-Universität Bochum for hospitality. 
now we have several expressions:

$$
\begin{aligned}
Z & =G / Q=G_{u} /\left(G_{u} \cap Q\right)=A /(A \cap Q)=B /(B \cap Q) \\
& =A^{0} /\left(A^{0} \cap Q\right)=B^{0} /\left(B^{0} \cap Q\right) .
\end{aligned}
$$

According to [W1, Prop. 3.1] there are just a few possibilities for a homogeneous almost-hermitian manifold $Z$ to have distinct expressions such as $G_{u} / L_{u}$ and $B^{0} /\left(B^{0} \cap L_{u}\right)$, where $G_{u}$ is the identity component of the group of all almosthermitian isometries, $G_{u}$ is simple, $L_{u}$ is the centralizer of a torus subgroup of $G_{u}$, and $B^{0} \varsubsetneqq G_{u}$ with $B^{0}$ connected. They are :

$(1.2) Z=P^{2 n-1}(\mathbb{C})=S U(2 n) / U(2 n-1)=S p(n) /(S p(n-1) \cdot U(1))$, complex projective space,

(1.3) $Z=S O(2 r+2) / U(r+1)=S O(2 r+1) / U(r)$, unitary structures on $\mathbb{R}^{2 r+2}$,

(1.4) $Z=S O(7) /(S O(5) \cdot S O(2))=G_{2} / U(2), 5$-dimensional complex quadric, and

(1.5) $Z=S O(8) /(S O(6) \cdot S O(2))=\left\{\operatorname{Spin}(7) / Z_{2}\right\} / U(3), 6$-dimensional complex quadric.

This applies in our situation because $L_{u}=G_{u} \cap Q$ is the centralizer of a torus subgroup of $G_{u}$, and $Z$ has a $G_{u}$-invariant hermitian metric.

Now return to the expression $Z=G / Q$. $G$ (and thus $G_{u}$ ) is simple. Let $A \varsubsetneqq G$ be a closed subgroup that is transitive on $Z$ and let $B$ be its maximal compact subgroup. We may assume $B=A \cap G_{u}$. Then $B \varsubsetneqq G_{u}, B^{0}$ is transitive on $Z$, and the expression $Z=G_{u} / L_{u}=B^{0} /\left(B^{0} \cap L_{u}\right)$ is given above. In each case the group $B^{0}$ is simple, so $A^{0}$ has Levi decomposition $A^{0}=A_{s s}^{0} A_{\text {rad }}^{0}$ into semisimple part and solvable radical, where $B^{0}$ is a maximal compact subgroup of $A_{s s}^{0}$. We run through the 4 possibilities listed above.

For $(1.2), G=S L(2 n ; \mathbb{C})$ and $B^{0}=S p(n)$. The semisimple Lie groups with maximal compact subgroup $S p(n)$ are $S p(n), S p(n ; \mathbb{C})$, the quaternionic linear group $S L(n ; \mathbb{H})$, and, for $n=4$, the real group $F_{4, C_{4}}$. But $F_{4}$ does not have a representation of degree 8 , in other words $F_{4} \not \subset G$, so now $A_{s s}^{0}$ is one of $\operatorname{Sp}(n), S p(n ; \mathbb{C})$ and $S L(n ; \mathbb{H})$. Each of them is irreducible on $\mathbb{C}^{2 n}$, so the unipotent radical of the algebraic hull of $A^{0}$ acts trivially on $\mathbb{C}^{2 n}$ and the center of the reductive part of $A^{0}$ acts by scalars. As $G$ acts effectively and by transformations of determinant 1 on $\mathbb{C}^{2 n}$ now $A_{s s}^{0}=A^{0}$, so $A^{0}$ is one of $S p(n), S p(n ; \mathbb{C})$ and $S L(n ; \mathbb{H})$. If $g \in G$ normalizes $A^{0}$, then some element $g^{\prime} \in g A^{0}$ centralizes $A^{0}$, because $A^{0}$ has no rational outer automorphism. As $A^{0}$ is irreducible on $\mathbb{C}^{2 n}$ now $g^{\prime}$ is scalar (and thus acts trivially on $Z$ ). Thus $A=A^{0} F$ where $F$ can be any subgroup of the center $\left\{e^{2 \pi i k / 2 n} I \mid 0 \leqq k<2 n\right\}$ of $G$.

For (1.3), $G=S O(2 r+2 ; \mathbb{C})$ and $B^{0}=S O(2 r+1)$. The semisimple Lie groups with maximal compact subgroup $S O(2 r+1)$ are $S O(2 r+1), S O(2 r+$ $1 ; \mathbb{C}), S O(1,2 r+1)$, and $S L(2 r+1 ; \mathbb{R})$. But $A_{s s}^{0}=S L(2 r+1 ; \mathbb{R})$ would give $S L(2 r+1 ; \mathbb{C}) \subset S O(2 r+2 ; \mathbb{C})$, so the respective dimensions would satisfy $4 r^{2}+4 r \leqq$ $2 r^{2}+3 r+1$, forcing $r=0$ and $Z=$ (point). Thu $\left\{A_{s s}^{0} \neq S L(2 r+1 ; \mathbb{R})\right.$. Now $A_{s s}^{0}$ is one of $S O(2 r+1), S O(2 r+1 ; \mathbb{C})$, and $S O(1,2 r+1)$. The last one acts irreducibly on $\mathbb{C}^{2 r+2}$, and there $A_{s s}^{0}=A^{0}$ as above. For the first two, recall that $S O(2 r+1)$ is absolutely irreducible on the tangent space $\mathfrak{s} o(2 r+2) / \mathfrak{s} o(2 r+1)$ of the sphere $S^{2 r+1}$, so $A_{\text {rad }}^{0}$ has Lie algebra reduced to 0 , and again $A_{s s}^{0}=A^{0}$. Now $A^{0}$ is one of $S O(2 r+1), S O(2 r+1 ; \mathbb{C})$, and $S O(1,2 r+1)$. If $g \in G$ normalizes $A^{0}$, then some

1 The author thanks the referee for a comment that improved and clarified his treatment of this $S L(2 r+1 ; \mathbb{R})$ case. 
element $g^{\prime} \in g A^{0}$ centralizes $A^{0}$, because $A^{0}$ has no rational outer automorphism. Thus either $A=A^{0}$ or $A / A^{0}$ has order 2 where $A$ is one of $O(2 r+1), O(2 r+1 ; \mathbb{C})$, and $S O(1,2 r+1) \cdot\{ \pm I\}$.

For $(1.4), G=S O(7 ; \mathbb{C})$ and $B^{0}=G_{2}$. The semisimple Lie groups with maximal compact subgroup $G_{2}$ are $G_{2}$ and its complexification $G_{2, \mathbb{C}}$. They are irreducible on $\mathbb{C}^{7}$ and have no rational outer automorphisms, so, as before, $A^{0}$ is either $G_{2}$ or $G_{2, \mathbb{C}}$, and if $g \in G$ normalizes $A^{0}$, then some element $g^{\prime} \in g A^{0}$ centralizes $A^{0}$. This forces $g^{\prime}$ to be central in $S O(7 ; \mathbb{C})$, so $g^{\prime}=1$ and $A=A^{0}$. Thus $A$ is either $G_{2}$ or $G_{2, \mathbb{C}}$.

Finally, (1.5) is obtained from the case $r=3$ of (1.3) by applying the triality automorphism, so it does not give us anything more.

In summary,

Theorem 1.6. Consider a complex flag manifold $Z=G / Q$. Suppose that $Z$ is irreducible, i.e., that $G$ is simple. Then the closed subgroups $A \subset G$ transitive on $Z, G_{u} \neq A \neq G$, are precisely those given as follows:

1. $Z=S U(2 n) / U(2 n-1)=P^{2 n-1}(\mathbb{C})$ complex projective $(2 n-1)$-space; $G=S L(2 n ; \mathbb{C})$ and $A=A^{0} F$ where $A^{0}$ is one of $S p(n), S p(n ; \mathbb{C})$ and $S L(n ; \mathbb{H})$, and $F$ is any subgroup of the center $\left\{e^{2 \pi i k / 2 n} I \mid 0 \leqq k<2 n\right\}$ of $G$. Here $F$ acts trivially on $Z$, so $A$ and $A^{0}$ have the same action on $Z$.

2. $Z=S O(2 r+2) / U(r+1)$, unitary structures on $\mathbb{R}^{2 r+2} ; G=S O(2 r+2 ; \mathbb{C})$ and $A=A^{0} F$ where $A^{0}$ is one of $S O(2 r+1), S O(2 r+1 ; \mathbb{C})$, and $S O(1,2 r+1)$, and where $F$ is any subgroup of the center $\{ \pm I\}$ of $G$. Here $F$ acts trivially on $Z$, so $A$ and $A^{0}$ have the same action on $Z$.

3. $Z=S O(7) /(S O(5) \cdot S O(2)), 5$-dimensional complex quadric; $G=S O(7 ; \mathbb{C})$ and $A$ is either the compact connected group $G_{2}$ or its complexification $G_{2, \mathbb{C}}$.

Picking out the cases where $A$ is a real form of $G$ we have

Corollary 1.7. Consider a complex flag manifold $Z=G / Q$. Suppose that $Z$ is irreducible, i.e., that $G$ is simple. Then the (connected) noncompact real forms $G_{0} \subset G$ transitive on $Z$ are precisely those given as follows:

1. $Z=S U(2 n) / U(2 n-1)=P^{2 n-1}(\mathbb{C})$ complex projective $(2 n-1)-$ space; $G=S L(2 n ; \mathbb{C})$ and $G_{0}$ is the quaternion linear group $S L(n ; \mathbb{H})$, which has maximal compact subgroup $S p(n)$.

2. $Z=S O(2 r+2) / U(r+1)$, unitary structures on $\mathbb{R}^{2 r+2} ; G=S O(2 r+2 ; \mathbb{C})$ and $G_{0}$ is the Lorentz group $S O(1,2 r+1)$, which has maximal compact subgroup $S O(2 r+1)$.

\section{The SOLUtion FOR FLAG MANIFOLDS IN GENERAL}

We complete the solution of the problem by reducing it to the case where $Z$ is irreducible.

Proposition 2.1. Decompose $G=\prod G_{i}$, the local direct product of complex connected simple Lie groups. Thus $Z=\prod Z_{i}$, the product of irreducible flag manifolds $Z_{i}=G_{i} / Q_{i}$ where $Q_{i}=Q \cap G_{i}$. Then $A^{0}=\prod A_{i}^{0}$ with $A_{i}^{0}=A^{0} \cap G_{i}$ and $B^{0}=\prod B_{i}^{0}$ with $B_{i}^{0}=B^{0} \cap G_{i}$. The groups $A_{i}^{0}$ and $B_{i}^{0}$ are connected, simple, and transitive on $Z_{i}$.

Proof. The solvable radical of $A^{0}$ is contained in a Borel subgroup of $G$, and thus has a fixed point on $Z$. It is normal in the transitive group $A^{0}$ so it fixes every point. Thus $A^{0}$ is semisimple. Similarly $B^{0}$ is semisimple. 
Let $\pi_{i}: G \rightarrow G_{i}$ denote the projection. The compact connected group $\pi_{i}\left(B^{0}\right)$ is transitive on $Z_{i}$. So it must be the compact real form $G_{u, i}=G_{i} \cap G_{u}$ of $G_{i}$ or one of the compact connected transitive groups described in (1.2), (1.3) or (1.4). (Recall that (1.5) is in fact a special case of (1.3).) In all cases, $\pi_{i}\left(B^{0}\right)$ is nontrivial and simple. Now $\pi_{i}$ annihilates all but one of the simple factors of $B^{0}$. Obviously no simple factor of $B^{0}$ is annihilated by every $\pi_{i}$. So now $B^{0}=\prod B_{\alpha}^{0}$ where the $B_{\alpha}^{0}$ are simple and where the index set $I$ for $G=\prod_{I} G_{i}$ is a disjoint union of subsets $I_{\alpha}$ with $B_{\alpha}^{0} \subset \prod_{i \in I_{\alpha}} G_{i}$. The proof of Proposition 2.1 is reduced to the case where $B^{0}$ (and thus also $A^{0}$ ) is simple, and there it is reduced to the proof that $G_{u}$ is simple.

We may now assume $B^{0}$ simple. Suppose that $G_{u}$ is not simple. Projecting to $G_{1} \times G_{2}$ we may assume $G=G_{1} \times G_{2}$. View the isomorphisms $\pi_{i}: B^{0} \cong \pi_{i}\left(B^{0}\right)$ as identifications. Denote $E_{i}=\pi_{i}\left(B_{\mathbb{C}}^{0}\right)$, the complexification of the image of $B^{0}$ in $G_{i}$. Denote $E_{u, 1}=\pi_{i}\left(B^{0}\right)$, the compact real form of $E_{i}$. Denote $P_{i}=E_{i} \cap Q_{i}$, the parabolic subgroup of $E_{i}$ that is its isotropy subgroup in $Z_{i}$, so $Z_{i}=E_{i} / P_{i}$. Now $B_{\mathbb{C}}^{0}=\left\{(e, e) \mid e \in E_{1}\right\}, B_{\mathbb{C}}^{0} \cap Q=\left\{(p, p) \mid p \in\left(P_{1} \cap P_{2}\right)\right\}$, and $Z=B_{\mathbb{C}}^{0} /\left(B_{\mathbb{C}}^{0} \cap Q\right) \cong$ $E_{1} /\left(P_{1} \cap P_{2}\right)$. In particular $P_{1} \cap P_{2}$ is a parabolic subgroup of $E_{1}$. Compute complex dimensions: $\operatorname{dim} E_{1}-\operatorname{dim}\left(P_{1} \cap P_{2}\right)=\operatorname{dim} B^{0}-\operatorname{dim}\left(B^{0} \cap Q\right)=\operatorname{dim} Z=$ $\operatorname{dim} Z_{1}+\operatorname{dim} Z_{2}=\left(\operatorname{dim} E_{1}-\operatorname{dim} P_{1}\right)+\left(\operatorname{dim} E_{1}-\operatorname{dim} P_{2}\right)$. On the Lie algebra level this says $\operatorname{dim} \mathfrak{e}_{1}=\operatorname{dim} \mathfrak{p}_{1}+\operatorname{dim} \mathfrak{p}_{2}-\operatorname{dim}\left(\mathfrak{p}_{1} \cap \mathfrak{p}_{2}\right)$, in other words $\mathfrak{p}_{1}+\mathfrak{p}_{2}=\mathfrak{e}_{1}$. As $\mathfrak{p}_{1} \cap \mathfrak{p}_{2}$ is a parabolic subalgebra of $\mathfrak{e}_{1}$ we have a Cartan subalgebra $\mathfrak{h}$ and a Borel subalgebra $\mathfrak{s}$ with $\mathfrak{h} \subset \mathfrak{s} \subset \mathfrak{p}_{1} \cap \mathfrak{p}_{2}$. In the root order such that $\mathfrak{s}$ is the sum of $\mathfrak{h}$ and the negative root spaces, no parabolic containing $\mathfrak{s}$ can contain the root space for the maximal root. This contradicts $\mathfrak{p}_{1}+\mathfrak{p}_{2}=\mathfrak{e}_{1}$. The contradiction proves $G_{u}$ simple and completes the proof.

Combining Proposition 2.1 with Theorem 1.6 we have

Theorem 2.2. Let $Z=G / Q$, the complex flag manifold, where $G$ is a complex connected semisimple Lie group acting with finite kernel on $Z$. Then the closed subgroups $A \subset G$ transitive on $Z$ are precisely those given as follows. Decompose $G=\prod G_{i}$ with $G_{i}$ simple, so $Z=\prod Z_{i}$ with $Z_{i}=G_{i} /\left(Q \cap G_{i}\right)$. Then $A=A^{0} F$ where $A^{0}=\prod A_{i}$ with $A_{i}=\left(A \cap G_{i}\right)^{0}$, and $A_{i}$ is equal to $G_{i}$, or to its compact real form $G_{u, i}$, or to one of the three types listed in Theorem 1.6 and $F$ is any subgroup of the center of $G$. Here $F$ acts trivially on $Z$, so $A$ and $A^{0}$ have the same action on $Z$.

Picking out the cases where $A$ is a real form of $G$ we have, as in Corollary 1.7,

Corollary 2.3. Let $Z=G / Q$, the complex flag manifold, where $G$ is a complex connected semisimple Lie group acting with finite kernel on $Z$. Then the (connected) real forms $G_{0} \subset G$ transitive on $Z$ are precisely those given as follows. Decompose $G=\prod G_{i}$ with $G_{i}$ simple, so $Z=\prod Z_{i}$ with $Z_{i}=G_{i} /\left(Q \cap G_{i}\right)$. Then $A=\prod A_{i}$ where $A_{i}=A \cap G_{i}$ either is the compact real form $G_{u, i}$ of $G_{i}$ or is one of the two types listed in Corollary 1.7 .

\section{REFERENCES}

[M] D. Montgomery, Simply connected homogeneous spaces, Proc. Amer. Math. Soc. 1 (1950), 467-469. MR 12:242c

[O1] A. L. Onishchik, Inclusion relations among transitive compact transformation groups. Trudy Moskov. Mat. Obšč. 11 (1962), 199-142. 
[O2] A. L. Onishchik, Topology of Transitive Transformation Groups, Johann Ambrosius Barth, Leipzig/Berlin/Heidelberg, 1994. MR 95e:57058

[W1] J. A. Wolf, The automorphism group of a homogeneous almost complex manifold. Trans. Amer. Math. Soc. 144 (1969), 535-543. MR 41:956

Institut für Mathematik, Ruhr-Universität Bochum, D-44780 Bochum, Germany

(Permanent address) Department of Mathematics, University of California, Berkeley, CALifornia 94720-3840

E-mail address: jawolf@math.berkeley.edu 